\title{
PSYCHOLOGIE PROJECTIVE ET AGRESSIVITÉ*
}

\author{
Jordi BACHS i COMAS**
}

\section{RESUMEN}

En este trabajo, a la luz de las aportaciones de Freud y Szondi, se examina el tema de la agresividad humana como un problema básicamente ético. La conciencia moral, en efecto, surge de la tensiön dialéctica entre conflictos pulsionales en dos procesos distintos, aunque paralelos y complementarios: en la relación triangular yo-padre-hermano (complejo de Cain) y en la otra relación, triangular también, yo-padre-madre (complejo de Edipo).

En una segunda parte, se propone el punto de vista de la psicología proyectiva y en especial los trabajos de Louis Corman, como un método indispensable para el estudio psicodinámico de la agresividad humana.

\section{$A B S T R A C T$}

This work, taking into account the contributions of Freud and Szondi, examines the theme of human agressivity as a basically ethical problem. Mo-

*Aquest treball va ser llegit en el 7è Congrés de la Societat Europea de Psiquiatria del Nen $i$ de l'Adolescent, celebrat a Lausanne (Suïssa) del 4 al 8 de juliol de 1983.

** Professor de "Psicodiagnòstic» i «Tècniques Projectives» del Departament de Psicologia Clínica de la Universitat Autònoma de Barcelona. 
ral conscience, in fact, emerges from dialectic tension of pulsional conflicts, in two different, though parallel and complementary processes: the triangular relations I-father-brother (Cain complex) and I-father-mother (Oedipus complex.

In the second part, the point of view of projective psychology, especially Dr. Louis Corman's work, is proposed as an indispensable method for the psychodynamic study of human agressivity.

\section{UN PROBLEME ETHIQUE}

Il y a déjà plus d'un demi siècle, dans son ouvrage Malaise dans la Civilisation, Freud posait ainsi la question du sort de l'espèce humaine: «le progrés de la civilisation saura-t-il, et dans quelle mesure, dominer les perturbations apportées à la vie en commun par les pulsions humaines d'agression et d'autodestruction?" Je me demande comment formulerait-il à notre époque ce qu'il y ajoutait ensuite: «les hommes d'aujord'hui ont poussé si loin la maîtrisse des forces de la nature qu'avec leur aide il leur est devenu facile de s'exterminer mutuellement jusqu'au dernier. Ils le savent bien, et c'est ce qui explique une bonne part de leur agitation présente, de leur malheur et de leur angoisse».

En rapportant ces mots de Freud, je voudrais souligner que le problème de l'agressivité humaine nous amène, tout compte fait, à celui du meurtre qui est un problème fondamental de l'éthique. Puis-je tuer? appelle pour reponse «je ne peux pas tuer». La répression de la tendance homicide est la condition même du surgissement de la société ainsi que de sa survie, J'ajouterai que la sphère de l'éthique est liée aussi au problème des affects et des sentiments. Outre l'interdit du meurtre, l'éthique se constitue par une regulation affective, celle des sentiments qui se rapportent au meurtre, tels la haine, la vengeance, le ressentiment. 
Je ne crois pas m'égarer dans un terrain qui ne concernerait pas la psychologie, si je me demande d'abord quels son les éléments psychiques qui, en se nouant, pourraient expliquer l'emergence et la structuration de la conscience éthique. En deuxième lieu, en descendant dans l'arène du concret, j'aimerais voir si la psychologie projective peut ouvrir, parmi d'autres disciplines paralleles, une voie de compréhension de l'agressivité de l'enfant.

\section{COMPLEXE DE GAIN ET COMPLEXE D’OEDIPE}

En me plaçant dans une perspective psychanalytique, je constate que du point de vue pulsionnel l'homme est un être amoral. Dès lors, la conscience ne súrgirait que dans le rapport dialectique de conflits pulsionnels au cours de deux processus distincts, mais paralleles et complémentaires: celui du complexe de Caïn et celui de l'Oedipe.

La question fondamentale, "puis-je tuer", est vecue négativement par Caïn et positivement par une autre figure biblique, Moïse, l'homme de loi. Moïse avait tué un jour. Il avait connu la terrible puissance de celui qui supprime la vie. Ensuite, ayant vecu le contrecoup réprobateur de son geste, il devient le Caïn positive et apporte la loi contre le meurtre. Plutôt que d'opposer deux polarités irreconciliables, la figure paradoxale de Moïse nous invite à considérer dans un rapport dialectique le crime et la loi, dans un continuum qui s'étale entre la tendance homicide et la reconnaissance de la loi.

Caïn est bien l'homme habité par la pulsion de mort. Mais on oublie parfois que, jaloux de son frère, il le tue pour avoir por lui tout seul l'amour du père. Pour expliquer donc le complexe de Caïn on ne peut pas oublier la coexistence conflictuelle d'Eros et Thanatos dans le triangle relationnelle moi-père-frère.

Le conflit est double. D'abord envers le père, décevant par son attitude bienveillante pour l'autre frère. Or, l'amour paternel seulement peut être atteint par la voie du renoncement, le deuil de l'aspiration libidinale d'un amour duel exclusif, qui débouche dans le fratricide. Porté par son désir de se faire reconnaitre par le père, Caïn est a mếme de renoncer à ses pulsions en acceptant et introjectant sa loi, «tu ne tueras point».

Deuxièmement, le conflit oppose Caïn à son frère, haï justement à cause du père. S'originant ainsi l'agressivité dans la loi externe du père, se retourne ensuite contre l'agresseur. Ce sont les reproches de conscience qui aboutissent, par un effort soucieux de réparation, à la resolution du double conflit. 
L'agressivité meurtrière est neutralisée. L'agresseur accepte le père comme modèle et dépositaire de la loi. Accepté à son tout par lui, et s'identifiant à lui, peut alors aimer son frère du même amour bienveillant. Le conflit s'évanouit ainsi dans la reconnaissance réciproque. La conscience éthique ne reléve donc pas de la seule économie libidinale, mais des forces en jeu dans le triangle relationnel, où la loi du père, la relation au frère (modifiant le lien libidinal tendant vers l'exclusivité) et l'image paternelle positive, permettent le dépassement du surmoi destructeur par l'intériorisation de la loi.

Parallèlement au complexe de Caïn, le complexe d'OEdipe constitue aussi un événement éthique parce qu'il libère l'enfant des attaches libidinales et l'insère dans la famille et la société. Il s'agit aussi des rapports conflictuelles, cette fois d'un triangle constitué par le père, la mère et l'enfant. Lé père, comme on sait, est celui qui fait éclater l'union de fusion affective de l'enfant à la mère, attirant vers lui l'aggressivité inhérante à l'amour de possession. Quand même, relié au père par une tendresse originaire, l'enfant est capable de renoncer à la démesure de son désir et à la violence meurtrière en assimilant la loi du père, germe de la conscience éthique. Ici encore, c'est le rapport dialectique de tendresse et violence qui fait éclater le conflit.

Je crois, dans cette perspective, qu'il faut envisager l'OEdipe comme une structure conflictuelle permanente (à l'oeuvre au sein de la famille et de la société) et pas du tout comme une excitation libidinale passagère de l'enfance. C'est ainsi que l'agressivité oedipienne, devenue pathogène par refoulement, peut se transposer sur toutes les figures substitutives du père ou se tourner contre le sujet dans des conduites d'autodestruction ou de culpabilité morbide. Par contre, médiatisée par la tendresse, elle reprend l'interdit paternel et donne origine à la conscience morale.

La resolution du conflit oedipien aboutit donc dans la reconnaissance de l'autre dans sa singularité. Ceci fait de l'homme un être éthique, en l'introduisant dans la constellation familiale et dans la société, de mème que le complexe de Caïn l'introduit, comme nous l'avons vu, dans la fratrie.

Le renoncement à la fusion affective fait de l'homme un être conscient du reel, de la loi, capable donc de vie éthique. La loi du père, en séparant l'enfant de la mère, creuse l'espace indispensable de la liberté, de l'existènce autonomme et du désir véritable, D'autre part, la reconnaissance du frère par la maîtrisse des tendances destructrices, instaure un être tolerant et bienveillant, capable de reconnaître l'autre en tant que personne. Ils s'éclairent dans ce contexte les mots de Levinas dans Totalité et infini: «la plenitude du bonheur est dans l'amour où le désir se creuse par la présence de l'autre». 


\section{ÉTUDE ET COMPREHENSION DE L'AGRESSIVITÉ DE L'ENFANT}

Après ces reflexions sur les figures mythiques d'OEdipe et Caïn, je voudrais esquiser maintenant le dernier volet de mon triptyque, en me référant à la psychologie projective dans un point tout à fait concret et pratique.

Cerner le cheminement pris par l'agressivité au cours du développement affectif individuel constitue sans doute un élément indispensable pour la connaissance structurale et dynamique de la personnalité de l'enfant et de l'adolescent. Et si l'agressivité, comme nous avons dit, peut devenir pathologique par refoulement, notre intérêt doit se porter non seulement sur les manifestations directement aggressives, mais aussi sur les formes masquées d'agressivité. En ce sens on sait, dẻpuis le Thematic Apperception Test, la valeur clinique des tecniques projectives dans l'étude de la personnalité profonde. A l'école de Murray, de Bellak (Children's Apperception Test) et spécialement de Gerald Blum dans son Blacky Pictures Test, le psychiatre français Louis CORMAN a élaboré, il y a déjà plus de vingt ans son test «Les Aventures de Patte Noire» (Test PN).

On sait que les tests dérivés du T.A.T. substituent les personnages humains des planches par des animaux familiers, dans le but de favoriser la projection à l'aide du symbolisme animal. De même que Blum dans son test Blacky, Corman présente une série d'images (19 exactement) qui racontent l'histoire d'un petit cochon, Patte Noire, qui est montré à l'enfant dans un frontispice avec d'autres cochons, dont il doit préciser librement le sexe, l'âge et la parenté.

Les données psychanalytiques des stades du développement psychosexuel ont présidé l'élaboration du test de Corman. Ainsi les différentes planches evoquent à travers le symbolisme animal des thèmes se rapportant au stade oral passif, stade oral actif, stade sadique-anal et finalement stade oedipien. L'enfant choisit librement, dans un premier moment du test, les planches avec lesquelles il élabore les aventures de Pattenoire. Dans un deuxième moment, il est invité à classer les images en deux piles, celles aimées et celles non-aimées en nous disant la raison de son choix affectif. Finalement il choisit pour chaque situation un personnage d'identification (ce personnage pouvant être personne). Cette méthode, dénommée par Corman, des "préférences-identifications" constitue une des originalités de l'auteur dans le but d'étudier d'une façon systématique les rapports entre pulsions et defenses. Sous des apparences naïves d'un petit cochon à qui arrivent beaucoup des choses, le sujet deploît symboliquement les aléas de sa propre histoire affective, vécue à des niveaux différents, soit dans le rapport dialecti- 
que entre sensualité et tendresse, entre amour de possession et amour dans le mode de l'être, soit au niveau des rapports affectifs tissés dans ses relations au père, à la mère, à la fratrie.

Notre expérience clinique de plusieurs années nous permet d'affirmer la valeur inestimable de ce test thématique pour l'etude psychodynamique de l'agressivité humaine. Certes, des images evocatrices du stade sadique-oral et sadique-anal, de même que du stade oedipien, permettent l'approche du complexe de Caïn et du complexe d'OEdipe, mais en fait n'importe quelle image, dans le contexte du vécu individuel, peut évoquer une situation à forte charge agressive.

L'utilisation correcte de ce test en clinique psychiatrique ou psychologique permet, du point de vue psychodynamique, l'étude et la compréhension de l'agressivité de l'enfant envers ses parents (rivalité oedipienne) ainsi qu'envers ses frêres et soeurs (rivalité fraternelle). Le rapport conflictuel entre pulsion et censure fait apparaitre l'importance clinique du refoulement de l'agressivité, de même que le rôle capital de certains mécanismes de défense du moi, grâce auxquels l'agressivité s'exprime de façon déguisée ou déplacée. La culpabilité, choc en retour de l'agressivité, source de nom. breux processus dépressifs et du masochisme moral de certains enfants, s'exprime également de façon dramatique dan le test PN. Le point de vue de la psychologie projective s'avoue donc, a mon avis, indispensable dans un approche pluridisciplinaire de l'agressivité de l'enfant. 


\section{Bibliographie}

Corman, Louis, Le Test PN. Manuel 1, P.U.F., 1972. Trad. cast., El Test PN. Manual primero, Herder, 1979.

-. Le Test PN. 2. Le Compexe d'OEdipe, P.U.F., 1973. Trad. Cast., El Test PN. El complejo de Edipo, Herder, 1981.

-. Le Test PN. 3. La règle d'investissement, P.U.F., 1976. Trad. cast., El Test PN. La regla de investimiento, herder; 1983.

-. Psychopatologie de la rivalité fraternelle, Dessart, 1970. Trad. cast., Psicopatología de la rivalidad fraterna, Herder, 1974.

Freud. S., Malaise dans la civilisation, P.U.F., 1971. Trad. cast., El malestar en la cultura, Obras completas, III, Biblioteca Nueva, 1968.

Klein, M. \& Riviere. J., L'amour et la haine, Petite Bibliotheque Payot, 1969.

Szondi. L., Cain y el cainismo en la historia universal, Biblioteca Nueva, 1975. L'homme Moïse à la lumière de l'analyse du destin; Revue de Psychologie et des Sciences de l'Education, 1971, 6, pp. 428-445.

-. Introduction à l'analyse du destin, Nawelaerts, 1972.

Vergote. A., La Psychanalyse, science de l'homme, Dessart, 1964.

Complexe d'OEdipe et complexe de Caïn, Revue de Psychologie et des Sciences de l'Edu. cation, 1971, 6, pp. 446-455. 